\title{
Similarity Between Stimuli: An Experimental Test of the Luce and Restle Choice Models ${ }^{I}$
}

\author{
Donald L. Rumelhart and James G. Greeno \\ The University of Michigan, Ann Arbor, Michigan 48104
}

\begin{abstract}
A set of choice alternatives was constructed to test Restle's (1961) hypothesis about the role of similarity between alternatives in choice behavior. The set contained names of three political figures, three actresses, and three athletes, with the intention of producing considerable similarity within subsets and minimal similarity between subsets. Subjects were asked to select the individual with whom they would prefer to spend an hour of conversation. Paired-comparison choice data from 234 college subjects were consistent with expectations based on Restle's model, and disconfirmed Luce's (1959) stronger model which does not take similarity into account. Measurements of rcsponsc strength for choosing the individual alternatives, and of perceived similarity between pairs of alternatives can be obtained using Restle's model, and the present test provides evidence for the validity of such measurements. Finally, Restle's model is shown to be indistinguishable from a modification of Thurstone's (1927) Case V, where the assumption of zero correlation is relaxed to permit nonnegative correlations between pairs of discriminal dispersions.
\end{abstract}

The belief that similarity between stimuli plays a nontrivial role in determining choice behavior is widely held today. However, little is known about the extent and the manner in which stimulus similarity influences choice behavior. Krantz (1964) demonstrated that similarity between stimuli tended to make choice probabilities more extreme than would otherwise be predicted. The hypothesis that similarity does play an important role in a paired comparison choice situation can best be illustrated by an example. Consider the following two choice alternatives:

$$
c \text {, a trip to California }
$$

Further assume that some subject desires each alternative equally and hence $P(c, f)=$ probability of choosing $c$ over $f=\frac{1}{2}$. Define the relation $j I k$, the subject is

\footnotetext{
${ }^{1}$ Some of the results of this investigation were reported at meetings of the Midwestern Psychological Association, Chicago, May, 1968. The data were collected and some of the analyses were carried out while the authors were at Indiana University.
} 
indifferent to alternatives $j$ and $k$, to mean that $P(j, k)=\frac{1}{2}$. A desirable property of such a relation would be transitivity, i.e., if $c I f$ and $f I a$ then $c I a$. If the subjects were to be presented with a third alternative:

$$
a \text {, a trip to California plus an apple, }
$$

then it would be reasonable to expect that $f I a$ and hence $c I a$ or that the subject would be indifferent as to whether or not he gained an apple. Some of the existing models in choice theory that ignore stimulus similarity would make this prediction. However, intuition suggests that $P(c, a)$ probably would be close to zero, at least for an individual who likes apples. This example presents a situation where the similarity is obvious and the comparison is simple. Is similarity a powerful enough variable such that it warrants consideration in a situation where the similarity is not blatant? It is this question to which this paper is directed.

Luce (1959) proposed a choice axiom from which a model of behavior is derived. The axiom states, "Let $T$ be the set of alternatives $(x, y, z, t, u, \ldots)$ and let $R$ be some appropriate subset of $T$ containing alternative $x$, for example, $R=(x, y, z)$. Then Luce's axiom states that $P(x ; T)=P(R ; T) P(x ; R)$ " (Atkinson, Bower \& Crothers, 1965 , p. 140). That is, the probability that $x$ is chosen from $T$ is equal to the probability that any element from $R$ is chosen from $T$ times the probability that $x$ is chosen from $R$. The axiom guarantees the existence of a real-valued scale for the alternatives. The scale values will be referred to as the strengths of the alternatives. Various other properties may be derived (See Atkinson, et al., 1965), but we will concern ourselves with the following two:

(1) the $v$-scale. There exists a nonnegative real-valued function on the set of alternatives, such that the probability that alternative $a$ is chosen over $b$ when only $a$ and $b$ are presented is

$$
P(a, b)=\frac{v(a)}{v(a)+v(b)},
$$

where $v(i)$ is the strength of alternative $i$.

(2) the product rule.

$$
\frac{P(a, b)}{P(\bar{b}, a)} \cdot \frac{P(b, c)}{P(c, b)} \cdot \frac{P(c, a)}{P(a, c)}=1 .
$$

This model does not consider the effect of stimulus similarity and hence the model is subject to the criticism mentioned above. Restle (1961) presented a choice model in which stimulus similarity was given a significant role. He assumed that choice alternatives are represented as sets of valued aspects. If an alternative shares valued aspects 
with another alternative, then the sets which represent those alternatives would intersect. In the case where no aspects are shared then there is no overlap between sets and Restle's model reduces to Luce's. In the general case:

$$
P(a, b)=\frac{v(a)-o(a, b)}{v(a)+v(b)-2 o(a, b)}
$$

where $v(i)$ is the measure of the set associated with alternative $i$ and $o(i, j)=$ measure of similarity between sets $i$ and $j$.

In the example mentioned at the beginning, Restle's model would say that the choice between a trip to California, and a trip to California plus an apple, would, in essence be a choice between an apple and nothing.

In an effort to determine the relative effects of stimulus similarity it was decided to compare the Luce and Restle choice models in a situation where stimulus similarity was believed to have an effect.

\section{Method}

Design and Materials. Each subject was required to make 36 pairwise choices. The stimuli were the names of 9 well-known personalities. The 36 choices were composed of all possible pairs formed from the stimuli. The set of stimuli consisted of the names of three politicians (L. B. Johnson, Harold Wilson, Charles DeGaulle), three athletes (Johnny Unitas, Carl Yastrzemski, A. J. Foyt), and Three movie stars, (Brigitte Bardot, Elizabeth Taylor, Sophia Loren).

Subjects. The subjects were 234 undergraduates taking introductory psychology courses at Indiana University in 1967. All of the subjects participating did so in partial fulfillment of their course requirements.

Procedure. Each subject was given a list of stimuli during a regularly scheduled class period. The size of the tested groups ranged from 10 to 120 . The subjects were instructed to choose the person with whom they would rather spend an hour discussing a topic of their choosing.

\section{REsults}

The product rule turned out to be a very convenient statistic to use in separating the two models. Restle's model predicts that the product rule should hold when the intersection between the three sets representing the stimuli is either empty or equal. (These are sufficient conditions, not necessary.) The similarity parameter $o(a, b)$ was assumed to be equal for all pairs in which $a$ and $b$ belonged to different subgroups. Therefore, Restle predicts that there are 27 triads for which the product rule should hold; these are the triads formed by selecting one member from each of the three 
subgroups. The remaining 57 triads were instances where the product rule might not hold.

For any triad of alternatives $a, b$, and $c$ for which the product rule holds, we should be able to account for the three observed choice frequencies $P(a, b), P(b, c)$, and $P(a, c)$ using just two parameters. For example, let

$$
w_{1}=\frac{v_{a}}{v_{b}}, \quad w_{\mathbf{2}}=\frac{v_{b}}{v_{c}} .
$$

Then, using the two peroperties stated earlier,

$$
P(a, b)=\frac{w_{1}}{1+w_{1}}, \quad P(b, c)=\frac{w_{2}}{1+w_{2}}, \quad P(a, c)=\frac{w_{1} w_{2}}{1+w_{1} w_{2}} .
$$

For each triad of alternatives, we estimated a pair of parameter values $w_{1}$ and $w_{2}$ that provided a minimum value of the goodness-of-fit chi square statistic, with theoretical frequencies calculated according to Eq. (4). An iterative computer search (Chandler, 1965) was used. If the tests were independent, then the null hypothesis (i.e., the product rule) would imply that the distribution of obtained chi square statistics should have the chi square distribution with one degree of freedom. The tests are not independent; however, the theoretical chi square distribution still provides a useful basis for comparison according to both Restle's and Luce's models. We might expect the 27 triads selected from separate subgroups to yield chi square statistics distributed as $\chi^{2}(1)$. According to Luce's model, but not Restle's, we might expect the remaining 57 triads to yield chi square statistics distributed as $\chi^{2}(1)$. Table 1 shows the results which indicate that Restle's model can successfully predict where the product rule should hold, and where it might fail.

TABLE 1

Distribution of $\chi^{2}$ under Product Rule

\begin{tabular}{ccc}
\hline$\chi^{2}$ values & Frequency & Frequency \\
\cline { 2 - 3 } & $\begin{array}{c}\text { Predicted to hold } \\
\text { by Restle and Luce }\end{array}$ & $\begin{array}{c}\text { Predicted to hold } \\
\text { by Luce }\end{array}$ \\
\hline $0-.064(20 \%)$ & 2 & 7 \\
$.065-.275(20 \%)$ & 4 & 6 \\
$.275-.708(20 \%)$ & 7 & 8 \\
$.708-1.64(20 \%)$ & 8 & 7 \\
$>1.64(20 \%)$ & 6 & 29 \\
& $\chi^{2}=4.30$ & $\chi^{2}=31.40$ \\
& $d f=4$ & $d f=4$ \\
& $p>.3$ & $p \ll 0.01$ \\
\hline
\end{tabular}


The two models were then fit directly to the data. Maximum likelihood estimates were obtained for all parameters using Chandler's (1965) iterative search routine. For Luce's model, there are eight parameters. There is a response strength $v(i)$ for each alternative but one must be set arbitrarily. We set $v(9)=1.0$. For Restle's model, we need the eight response strengths plus nine overlap parameters, one for each pair of alternatives taken from a single subset. It seemed reasonable to expect that the choice alternatives would have different strengths for males and females. Therefore, in addition to analyzing all of the data combined (Pooled) the males and females were analyzed separately. It was assumed throughout that the overlap between stimuli from different subgroups was equal.

A summary of these results is shown in Table 2. The first column of estimates is based on only those data for which the overlap between sets was equal, i.e., the pairs where alternatives were in different subsets. There were 27 data points and eight estimated parameters, giving 19 degrees of freedom. Recall under this condition Restle's model is mathematically identical to Luce's model. The fit of the models to these data is satisfactory.

The second column represents the parameter estimates made for Restle's model when all of the 35 data points were used. Each overlap parameter was restricted to be greater than zero and less than the minimum of the two sets to which it applied. This restriction accounts for the slight differences in the chi square statistics when comparing column 1 and column 2. If this restriction were removed, the two columns would have identical chi square values and the $v(i)$ 's would agree. This is the case since for each new data point included, a parameter is added.

Column 3 represents the parameter estimates yielded for Luce's model when all of the data are fit. It is particularly important to note the large increase in the chi square statistic. Since Luce's model is a special case of Restle's, each difference between chi square statistics in columns 2 and 3 tests Luce's model as a null hypothesis against Restle's model as the alternative. If Luce's model were correct, these statistics would be distributed as $\chi^{2}(9)$. Each of the cells in column 3 yields a chi square statistic sufficiently large to reject Luce's model. However, Luce's model before the data from the similar stimuli were added, fit the data quite well (column 1). If only the data from the similar stimuli are considered, the goodness-of-fit for Iuce's model is

$$
\begin{array}{lll}
\text { Pooled, } \quad \chi^{2}=56.50 & d f=9 & p=\text { nil } \\
\text { Males, } \quad \chi^{2}=33.15 & d f=9 & p<.006 \\
\text { Females, } \chi^{2}=48.82 & d f=9 & p=\text { nil. }
\end{array}
$$

This is strong evidence in support of the hypothesis that similarity plays a significant role in choice behavior. Furthermore, the good fit of Restle's model to the data indicates that a consideration of similarity such as proposed by Restle is appropriate. Pairwise predictions for the models as well as observed values appear in Table 3. 


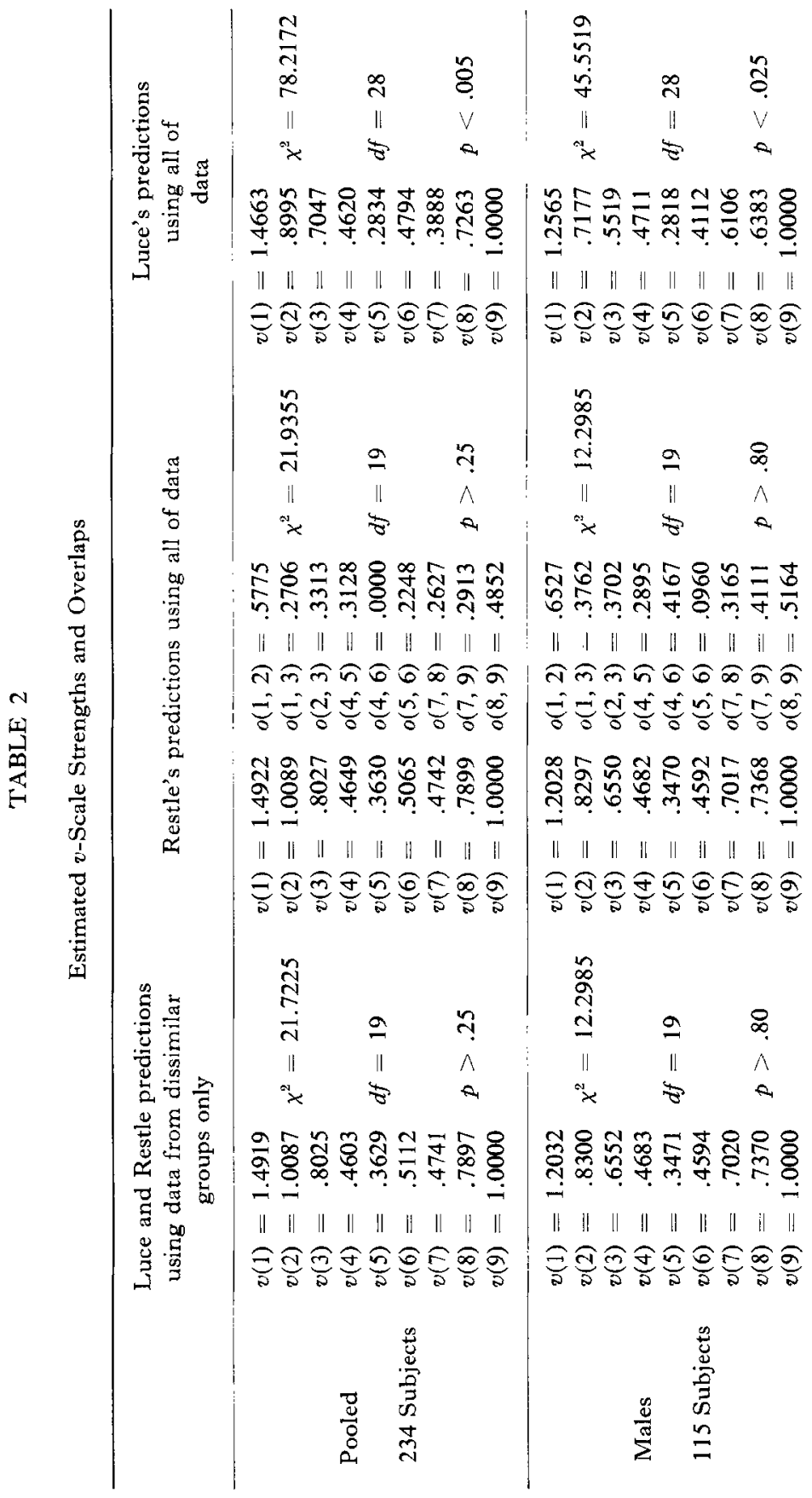




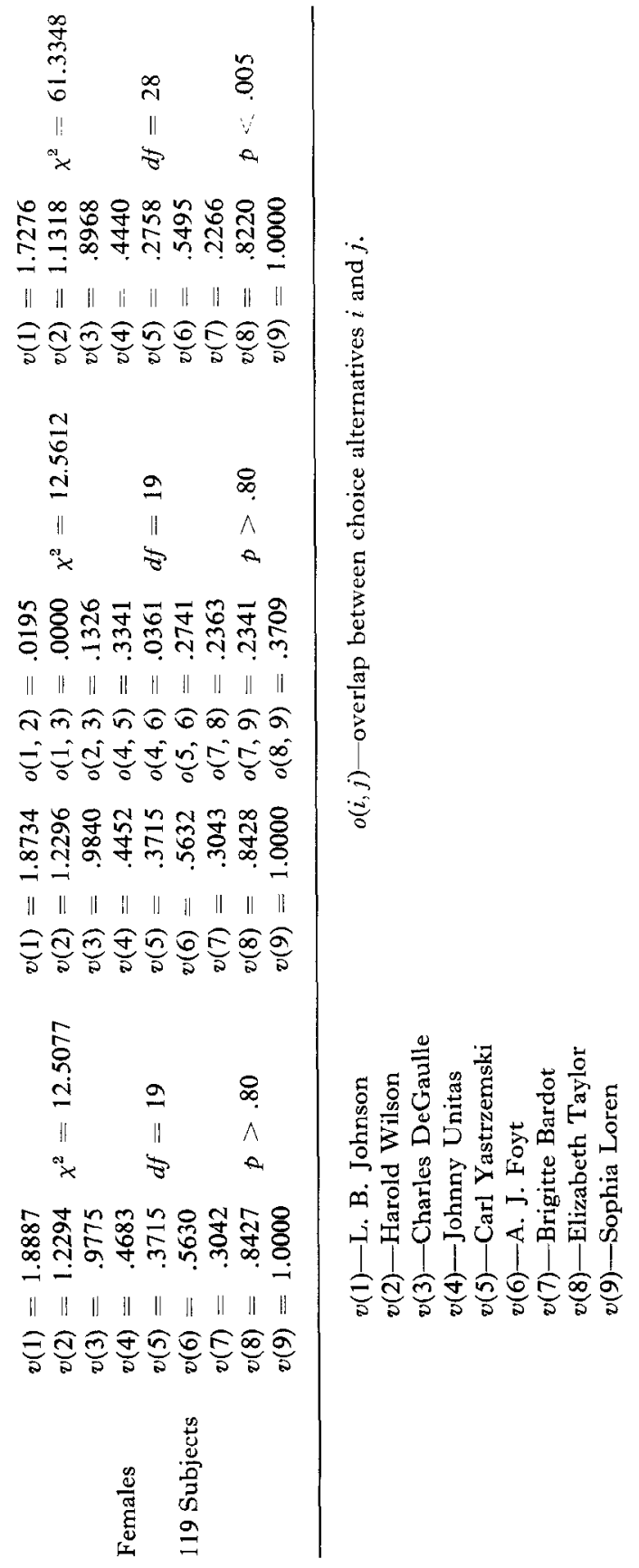


Some caution should be used in interpreting the above results. Both Luce and Restle assume that their models apply to individual choice behavior, and all statistics in this experiment were obtained by averaging across subjects. The pooling of data across subjects would have no effect if the following condition were satisfied:

Let $v_{i}(j)=$ the strength of alternative $j$ for subject $i$, then $v_{i}(j)=v_{k}(j)$ for all $j$ and $k$. This condition is rarely if ever met. This is certainly not met for the male and female subgroups of the data. $\left(\chi^{2}-205.06, d f-36, p==\right.$ nil for Restle's model and $\chi^{2}=179.32, d f=36, p=$ nil for Luce's model). The fact that the models continue to fit much of the existing data is evidence for the robustness of the models with respect to the assumption. Further empirical and statistical research is needed to clarify the extent and nature of the distortions in paired-comparison data caused by individual differences.

\section{Discussion}

We believe that our investigation takes a meaningful step toward understanding choice behavior. We have shown that stimulus similarity can influence choice behavior to such an extent that some existing models are incapable of making accurate predictions. We have also shown one model which is capable of handling stimulus similarity and we take our results as tentative support for the kind of choice mechanism proposed by Restle. Furthermore, if one is willing to accept Restle's model as a reasonable approximation, then we have succeeded in not only measuring the strengths of the stimuli but also measuring the pairwise similarities between some of the stimuli. Hence, we feel that the Restle model could be used as a basis of a measuring tool to experimentally estimate the similarity between stimuli.

\section{Restle's Model in ReLation to Ohers}

Tversky (1967) has proposed a model which is very similar to that of Restle's. Tversky hypothesized that given two stimuli $a$ and $b$, one will possess some advantages over the other and vice versa. Let $A_{a b}$ be the measure of the advantages of $a$ over $b$, then $A_{u b} \geqslant 0$ and $A_{b a} \geqslant 0$. Formally his theory is represented as

$$
P(a, b)=\frac{A_{a b}^{\theta}}{A_{a b}^{\theta}+A_{b a}^{\theta}} .
$$

If $\theta=1$, then Tversky's model is equivalent to Restle's.

We have already pointed out the relationship between Restle's model and Luce's. Earlier work (Luce and Suppes, 1965) has shown that Luce's model is indistinguishable 
from Thurstone's case $V$ (Thurstone, 1927) if Thurstone's assumption of normally distributed variables is replaced by the logistic cumulative function. Even with the normal assumption Thurstone's theory is quite close to Luce's in most cases (Burke \& Zinnes, 1965; Hohle, 1966; Luce \& Galanter, 1963). This brings up the interesting question as to the relationship between Restle's theory and Thurstone's. First, we examine Thurstone's theory in more detail.

Thurstone postulated that the stimuli to be compared could be mapped onto a psychological scale. In order to account for the fact that individuals are occasionally inconsistent in making choices, he assumed that the process of comparing two stimuli (discriminal process) was not a constant process, but rather a continually changing process depending on a discriminal distribution which could be represented for stimulus $i$ on the scale as being normally distributed about a mean $S_{i}$ and with variance $\sigma_{i}^{2}$.

These assumptions led to the formulation of his law of comparative judgment as follows:

$$
S_{i}-S_{j}=X_{i j} \sqrt{\sigma_{i}^{2}+\sigma_{j}^{2}-2 r_{i j} \sigma_{i} \sigma_{j}} \text {, where }
$$

$\chi_{i j}$ is the normal deviate corresponding to the proportion of times $i$ is judged greater than $j$, and $r_{i j}$ is the correlation between the discriminal processes for stimuli $i$ and $j$.

The law is never used in this form since there are always more unknowns than equations, and hence no solutions can be obtained. The most popular form of his law in his Case $\mathrm{V}$ in which simplifying assumptions are made to bring it to the form:

$$
S_{i}-S_{j}=c X_{i j}
$$

For a complete discussion see Torgerson (1958).

We assert that Restle's model is indistinguishable from Thurstone's general model if the logistic is substituted for the normal distribution and the following restrictions are met:

$$
\begin{array}{ll}
\text { (1) } \sigma_{i}{ }^{2}=\sigma_{j}{ }^{2} & \text { for all } i \text { and } j \text {; } \\
\text { (2) } \quad 0 \leqslant r_{i j} \leqslant 1 & \text { for all } i \text { and } j .
\end{array}
$$

Without loss of generality and to set the scale for Thurstone's model let $2 \sigma^{2}=1$. This yields as Thurstone's model: $S_{i}-S_{j}=X_{i j}\left(1-r_{i j}\right)^{\mathbf{1} / \mathbf{2}}$.

The logistic form of Thurstone's model can be expressed as

$$
P(i, j)=\left[1+e^{-\left(S_{i}-S_{j}\right) / c_{i j}}\right]^{-1} \quad \text { where } \quad c_{i j}=\left(1-r_{i j}\right)^{1 / 2} .
$$


Thus

$$
\frac{P(i, j)}{P(j, i)}=\frac{e^{S_{i} / c_{i j}}}{e^{S_{i} / c_{i j}}} .
$$

TABLE 3

Observed and Predicted Proportions of Choice of Row Stimuli rather than Column Stimuli

\begin{tabular}{|c|c|c|c|c|c|c|c|c|c|}
\hline & 1 & 2 & 3 & 4 & 5 & 6 & 7 & 8 & 9 \\
\hline \multirow{3}{*}{1} & Observed & $.680^{a}$ & $.697^{a}$ & .748 & .782 & .765 & .739 & .684 & .607 \\
\hline & Restle & .680 & .697 & .763 & .804 & .747 & .759 & .654 & .599 \\
\hline & Modified & .680 & .697 & .700 & .806 & .751 & .762 & .651 & .597 \\
\hline \multirow{3}{*}{2} & & & $.590^{a}$ & .700 & .735 & .684 & .667 & .521 & .521 \\
\hline & & & .590 & .685 & .735 & .666 & .680 & .561 & .502 \\
\hline & & & .590 & .698 & .740 & .677 & .689 & .567 & .510 \\
\hline \multirow{3}{*}{3} & & & & .620 & .671 & .590 & .598 & .521 & .513 \\
\hline & & & & .633 & .689 & .613 & .629 & .504 & .445 \\
\hline & & & & .650 & .695 & .627 & .640 & .513 & .457 \\
\hline \multirow{3}{*}{4} & & & & & $.752^{a}$ & $.492^{b}$ & .530 & .368 & .261 \\
\hline & & & & & .752 & .479 & .495 & .371 & .317 \\
\hline & & & & & .752 & .476 & .490 & .363 & .311 \\
\hline \multirow{3}{*}{5} & & & & & & $.329^{a}$ & .406 & .308 & .261 \\
\hline & & & & & & .329 & .434 & .315 & .266 \\
\hline & & & & & & .329 & .441 & .317 & .268 \\
\hline \multirow{3}{*}{6} & & & & & & & .573 & .393 & .303 \\
\hline & & & & & & & .517 & .391 & .336 \\
\hline & & & & & & & .514 & .386 & .332 \\
\hline \multirow{3}{*}{7} & & & & & & & & $.286^{a}$ & $.205^{\alpha}$ \\
\hline & & & & & & & & .286 & .205 \\
\hline & & & & & & & & .286 & .205 \\
\hline \multirow{3}{*}{8} & & & & & & & & & $.372^{a}$ \\
\hline & & & & & & & & & .372 \\
\hline & & & & & & & & & .372 \\
\hline 9 & & & & & & & & & \\
\hline
\end{tabular}

a All entries in these cells should be equal if argument in text is correct.

${ }^{b}$ All entries in this cell would have agreed if both models had not been on boundary of parameter space, due to reversal in data. 
Similarly Restle's model yields $P(i, j) / P(j, i)=[v(i)-o(i, j)] /[v(j)-o(i, j)]$. Setting the two equations equal yields:

$$
\frac{v(i)-o(i, j)}{v(j)-o(i, j)}=\left[\frac{e^{S_{i}}}{e^{S_{j}}}\right]^{1 / c_{i j}}
$$

The two models will be identical if we set $v(i)=e^{S_{i}}$ for all $i$, and

$$
C_{i j}=\frac{\log [v(i) / v(j)]}{\log \{[v(i)-o(i, j)] /[v(j)-o(i, j)]\}} \quad \text { for all } i \text { and } j^{2}
$$

The argument given above deals with Thurstone's model modified to assume the logistic rather than the normal distribution. The question remains whether Thurstone's model with equal variance normal distributions and nonzero correlations yields predictions that are approximately equal to those obtained from Restle's model. The fact that Thurstone's Case $\mathrm{V}$ is practically indistinguishable from Luce's model suggests a positive answer. To check this in our data, we obtained minimum chi square estimates of the parameters of Thurstone's model, and obtained the predictions shown as the third entry in each cell of Table 3. As can be seen, Restle's and Thurstone's models make very similar predictions.

\section{SuMmary}

In summary it has been shown that the generalization of Luce's model that yields Restle's is equivalent to a generalization of Thurstone's model with the logistic assumption in which correlations between discriminal dispersions are free parameters (we must restrict $r_{i j}$ to be nonnegative). Furthermore, if we use the normal distribution instead of the logistic as is usually done, the differences between Restle's and Thurstone's predictions will probably be insignificant. Hence, the only reason for choosing one model over the other appears to be convenience.

\section{REFERENCES}

Atkinson, R. C., Bower, G. H., AND Crothers, E. J. An introduction to mathematical learning theory. New York: Wiley, 1965.

Burke, C. J., AND Zinnes, J. L. A paired comparison of pair comparisons. Journal of Mathematical Psychology, 1965, 2, 53-76.

Chander, J. P. Subroutine Stepit. Program QCPE 66. Quantum Chemistry Program Exchange, Indiana University, Bloomington, Indiana, 1965.

2 The form of this argument was suggested by the reviewer. 
HoHLE, R. H. An empirical evaluation and comparison of two models for discriminability scales. Journal of Mathematical Psychology, 1966, 3, 174-183.

Krantz, D. H. The scaling of small and large color differences. Unpublished doctoral dissertation, University of Pennsylvania, 1964.

LuCE, R. D. Individual choice behavior: a theoretical analysis. New York: Wiley, 1959.

Luce, R. D., AND Galanter, E. Discrimination. In R. D. Luce, R. R. Bush, and E. Galanter (Eds.), Handbook of mathematical psychology. Vol. 1. New York: Wiley, 1963. Pp. 191-244.

Luce, R. D., AND Suppes, P. Preference, utility, and subjective probability. In R. D. Luce, R. R. Bush, and E. Galanter (Eds.), Handbook of mathematical psychology, Vol. 3. New York: Wiley, 1965. Pp. 249-410.

Restle, F. Psychology of judgment and choice. New York: Wiley, 1961.

Thurstone, L. L., A law of comparative judgment. Psychology Review, 1927, 34, 273-286.

Torgerson, W. S. Theory and methods of scaling. New York: Wiley, 1958.

Tversky, A. Advantage theory: a study of value and choice. Unpublished manuscript, Hebrew University, 1967.

Received: February 26, 1970 